\title{
La energía eólica en la producción de electricidad en España ${ }^{1}$
}

\author{
Cayetano Espejo Marín² y Ramón García Marín ${ }^{3}$
}

\section{RESUMEN}

España es uno de los países del planeta que más ha apostado por las energías renovables para la producción de electricidad durante la primera década del siglo XXI. La energía eólica ha sido la que más se ha desarrollado, por la presencia de vientos que hacen viable su explotación, por las políticas de fomento de todas las administraciones, y por la existencia de un sector industrial español muy innovador y competitivo, cuya actividad tiene un carácter global.

Palabras clave: Energía eólica, política energética, industria innovadora.

\begin{abstract}
Over the first decade of the 21st century, the Spanish governments have outstandingly pledged their commitment to the development of renewable energies. Wind energy has actually enjoyed the leading development in this respect, in connection with the presence of regular winds that make its exploitation feasible. Additional factors contributing to its production are: the application of policies encouraging them and the existence of an innovative and competitive industrial sector, with global-ranging activities.
\end{abstract}

Key words: Wind energy, energy policy, innovative industries

1 Artículo recibido el 19 de julio de 2011, aceptado el 15 de enero de 2012 y corregido el 5 de marzo de 2012.
2 Departamento de Geografía, Universidad de Murcia (España). E-mail: cespejo@um.es

3 Departamento de Geografía, Universidad de Murcia (España). E-mail: ramongm@um.es 
Las energías renovables han evolucionado en España hacia una participación creciente en el sistema energético, que se evidencia en la cobertura de la demanda, expresada tanto en términos de energía primaria como final. Esta situación ha experimentado un significativo aumento a partir del año 2005, en el que aportan el $6,1 \%$ del consumo de energía primaria, frente al 13,2\% en el año 2010.

En el año 2010 las energías renovables han incrementado su participación en la matriz energética española hasta alcanzar el $13,2 \%$ de la energía final, casi un punto por encima del $12,3 \%$ que representaron en 2009, por lo que se sitúa a España en una buena posición para alcanzar el objetivo de la Unión Europea de que en 2020 el 20\% del consumo energético proceda de energías renovables. El desarrollo de las tecnologías renovables ha sido mayor en el ámbito eléctrico, ya que en 2010 representan el 32,3\% de la generación total, con un incremento de más de 7 puntos sobre el año anterior y 2,9 puntos por encima del objetivo fijado en el Plan de Energías Renovables 2005-2010. Entre la producción de energía eléctrica con fuentes renovables destaca el papel desempeñado por la generación de la eólica e hidroeléctrica, que en 2010 aporta el $16 \%$ y $14,8 \%$ del total respectivamente, el $81,7 \%$ de toda la producción eléctrica renovable. En 2010, la energía eólica se consolida como la primera fuente renovable, por delante de la hidroeléctrica que tradicionalmente ha encabezado la producción eléctrica renovable en España (Espejo y García, 2010).

La producción bibliográfica sobre la energía eólica en España se ha centrado preferentemente en los siguientes aspectos: la distribución territorial de la potencia instalada, el desarrollo de la industria eólica, las políticas de fomento de las energías renovables y dentro de estas de la eólica, y el impacto de los aerogeneradores sobre el paisaje.

Espejo (2004) es autor del primer estudio geográfico de la energía eólica en España, en él analiza las características que definen a este sector, desde la distribución territorial de los vientos como recurso imprescindible, hasta los impactos que sobre el medio ambiente provoca esta actividad. Con posterioridad se dan dedicado dos trabajos siguiendo esta metodología a dos de las regiones españolas con mayor potencia eólica instalada: Castilla y León (Baraja y Herrero, 2010) y CastillaLa Mancha (Espejo, 2010). También se ha analizado el papel de la energía eólica como generadora de empleo en Navarra (Pintor et al., 2006) y los efectos económicos de esta energía en Aragón (Simón et al., 2009).

Sobre la industria dedicada a la energía eólica y su contribución al desarrollo rural destaca el trabajo de Martínez et al. (2002), y de la expansión internacional de Gamesa, una de las principales industrias eólicas del planeta, dan buena cuenta las publicaciones de López (2007) y López-Vico (2008).

El gran desarrollo de la energía eólica en España ha sido posible gracias a la existencia de unas políticas muy favorables, tal y como se analizan en las publicaciones de López dedicadas a la regulación y autorización de los parques eólicos (2008) y al apoyo de la energía eólica en España (2009).

El impacto de las instalaciones eólicas sobre el paisaje es también motivo de preocupación e investigación por parte de varios autores (Frolova y Pérez, 2008), (Mérida et al., 2009), (Pérez et al., 2009), (Frolova, 2010), (Baraja y Herrero, 2010). Aunque no hay todavía instalados parques eólicos marinos, se manifiesta un interés por su acertada ordenación territorial y los posibles impactos que puedan tener sobre los paisajes (Pérez, 2009, Pérez, 2010).

También en otras publicaciones se ha expuesto que la energía eólica, además de contribuir al desarrollo rural, también genera el rechazo de grupos conservacionistas y asociaciones vecinales por el impacto paisajístico que genera (Galdós y Madrid, 2009).

Reseñable también es el artículo que analiza el papel de las energía eólica en el proceso de transición hacia un nuevo sistema energético, más eficiente y diversificado y como una mejor gestión del territorio, donde el uso de los Sistemas de Información Geográfica constituye una herramienta fundamental para contribuir a acelerar la implantación de un modelo energético caracterizado por la sostenibilidad (Domínguez et al., 2010). 
El desarrollo reciente de la energía eólica en España ha convertido al país en uno de los pioneros a escala planetaria en la implantación masiva de esta tecnología de producción eléctrica. De ahí el interés por exponer los elementos que caracterizan al sector eólico en España y las razones que explican su expansión.

Este artículo tiene como objetivo el análisis desde el punto de vista geográfico, y por tanto territorial, de la energía eólica en España. Para ello se presenta la evolución de la potencia instalada y de la producción de energía eólica, para ver su papel en la producción total eléctrica; la distribución regional de la capacidad productiva; la localización de la industria dedicada a la fabricación de componentes, a la instalación y al mantenimiento de las instalaciones eólicas; también se exponen las directrices que han hecho posible la implantación y crecimiento de la energía eólica dentro de la Política de Fomento de las Energías Renovables en España; y por último, se señala la necesidad del aumento de la interconexión eléctrica de España con Europa para un óptimo aprovechamiento de la producción de las energías renovables.

\section{La energía eólica en la producción eléctrica española}

No hay país en el mundo que no haga suyo el objetivo que satisfacer, al menos en parte, sus necesidades de energía mediante el aprovechamiento de fuentes renovables. La energía eólica, basada en el aprovechamiento del viento, es la energía que más está destacando por la implantación conseguida y porque, en base a la reducción constante de sus costes, podrá competir con otras fuentes convencionales de energía eléctrica, extendiendo su presencia futura en todos los países del mundo (López, 2004).

España es uno de los países que han experimentado un mayor desarrollo industrial y tecnológico en el sector eólico dedicado a la producción de electricidad. La potencia instalada ha pasado de tener un carácter testimonial a finales del siglo pasado, con 2 megavatios (MW) en 1990 y 98 MW en 1995, a una sumar una capacidad 20.676 MW en 2010. Esta cifra sitúa a España en cuarto lugar en el planeta en cuanto a potencia instalada, detrás de China, Estados Unidos y Alemania (Cuadro № 1 ). 
Cuadro $N^{\circ} 1$

Evolución de la potencia instalada en los principales países, 2006-2010 (MW)

\begin{tabular}{|l|r|r|r|r|r|r|}
\hline País & \multicolumn{1}{c|}{2006} & \multicolumn{1}{c|}{2007} & \multicolumn{1}{c|}{2008} & \multicolumn{1}{c|}{2009} & \multicolumn{1}{c|}{2010} & \multicolumn{1}{|c|}{$\begin{array}{r}\text { Incremento } \\
2006-2010 \%\end{array}$} \\
\hline China & 2.599 & 5.912 & 12.210 & 25.810 & 44.733 & $1.621,2$ \\
Estados Unidos & 11.575 & 16.823 & 25.237 & 35.159 & 40.180 & 247,1 \\
Alemania & 20.622 & 22.247 & 23.897 & 25.777 & 27.214 & 32,0 \\
España & 11.586 & 15.104 & 16.689 & 19.149 & 20.676 & 78,5 \\
India & 6.270 & 7.850 & 9.587 & 11.807 & 13.066 & 108,4 \\
Italia & 2.123 & 2.726 & 3.736 & 4.850 & 5.797 & 173,1 \\
Francia & 1.567 & 2.455 & 3.404 & 4.574 & 5.660 & 261,2 \\
Reino Unido & 1.963 & 2.389 & 3.195 & 4.092 & 5.204 & 165,1 \\
Canadá & 1.460 & 1.846 & 2.369 & 3.319 & 4.008 & 174,5 \\
Portugal & 1.716 & 2.130 & 3.862 & 3.357 & 3.898 & 127,2 \\
Dinamarca & 3.136 & 3.125 & 3.163 & 3.465 & 3.752 & 19,6 \\
Japón & 1.309 & 1.528 & 1.880 & 2.083 & 2.304 & 76,0 \\
Países Bajos & 1.559 & 1.747 & 2.235 & 2.223 & 2.237 & 43,5 \\
Suecia & 571 & 831 & 1.067 & 1.448 & 2.163 & 278,8 \\
Resto de países & 3.066 & 7.214 & 8.372 & 12.653 & 15.738 & 413,3 \\
Total mundial & 71.122 & 93.927 & 120.903 & 159.766 & 196.630 & 176,5 \\
\hline
\end{tabular}

Fuente: The European Wind Energy Association \& World Wind Energy Association, 2010.

Este desarrollo ha sido posible por la disponibilidad de zonas con estimable potencial eólico, por las políticas de fomento de las energías renovables y por el despegue de una próspera industria que ha creado decenas de miles de empleos, en industrias muy repartidas por todo el territorio peninsular español. Además, al tratarse de una energía limpia, la energía eólica aporta numerosas ventajas medioambientales (Espejo, 2004).
En la primera década de este siglo la potencia eólica instalada en España se ha multiplicado por 8,7; cifra ligeramente inferior a lo que ha supuesto el incremento en la producción de electricidad. De ahí que su aportación a la cobertura de la demanda de electricidad Ilegue en la actualidad al 16\% (Cuadro № 2). 
Cuadro $\mathrm{N}^{\circ} 2$

Evolución anual de la potencia instada y electricidad producida con energía eólica, 2000-2010

\begin{tabular}{|c|r|r|r|}
\hline Año & \multicolumn{2}{|c|}{$\begin{array}{c}\text { Potencia } \\
\text { MW }\end{array}$} & $\begin{array}{c}\text { Producción } \\
\text { GWh }\end{array}$ \\
\hline 2000 & 2.365 & 4.668 & $\begin{array}{c}\text { Porcentaje de cobertura } \\
\text { de la demanda }\end{array}$ \\
2001 & 3.530 & 6.930 & 2,28 \\
2002 & 5.026 & 9.257 & 3,19 \\
2003 & 6.169 & 11.720 & 4,30 \\
2004 & 8.460 & 15.744 & 5,05 \\
2005 & 10.011 & 20.520 & 6,45 \\
2006 & 11.586 & 22.736 & 8,12 \\
2007 & 15.104 & 27.223 & 8,64 \\
2008 & 16.689 & 31.545 & 9,98 \\
2009 & 19.149 & 36.188 & 11,52 \\
2010 & 20.676 & 42.702 & 13,88 \\
\end{tabular}

Fuente: Asociación Empresarial Eólica, Red Eléctrica de España y Comisión Nacional de Energía, 2010.

En 2010 la energía eólica superó en varias ocasiones máximos históricos de potencia instalada, de energía horaria y de energía diaria. El 9 de noviembre se registró el último récord de energía diaria con 315.258 megavatios/hora (MWh), producción que permitió cubrir el $46 \%$ de la demanda de ese día. Asimismo, en febrero se produjo un máximo mensual que cubrió el $21 \%$ de la demanda de ese mes. Sin embargo, la variabilidad que caracteriza a esta energía ha dado lugar a situaciones extremas, como la producida el 9 de noviembre (3,35 horas) en la que el 54\% de la demanda fue cubierta por esta energía, mientras que el día 26 de junio a las 10,32 apenas aportó el $1 \%$. Por otro lado, la fuerte eolicidad del primer trimestre del año obligó a llevar a cabo ciertas limitaciones de producción durante algunas horas de demanda valle, lo que ha llevado a una pérdida cercana al 0,6\% del producible anual (Red Eléctrica de España, 2010).

Para hacer frente a las singularidades de las energías renovables, España cuenta desde 2006 con el Centro de Control de Energías Renovables (Cecre), una iniciativa pionera puesta en marcha por Red Eléctrica de Es- paña. Es el primer centro del mundo donde se controla y gestiona la generación de los productores de energías renovables, principalmente eólicos. Permite integrar en el sistema eléctrico la mayor cantidad de energía renovable posible manteniendo los niveles de calidad y garantizando la seguridad del suministro. A través de 23 centros de control, que actúan como interlocutores, el Cecre recibe cada 12 segundos información en tiempo real de cada instalación sobre el estado de la conexión a la red, la producción y la tensión en el punto de conexión. Estos datos son utilizados por sofisticadas herramientas que permiten comprobar si el total de generación de las energías renovables puede integrarse en cada instante en el sistema eléctrico sin afectar a la seguridad del suministro. Para anticiparse a las posibles incidencias que puedan surgir con las energías renovables, el Cecre analiza constantemente el escenario actual y prevé las medidas de operación necesarias para que el sistema se mantenga en un estado seguro. Este Centro de Control de Energías Renovables tiene un papel determinante en un sistema tan poco interconectado como el español. 
En 2010 la energía eólica se consolida como la tercera tecnología del sistema eléctrico español al haber alcanzado una producción de 42.702 Gigavatios/hora (GWh), solo superada por las centrales térmicas de gas de ciclo combinado (64.637 GWh) y las nucleares (61.787 GWh). La producción eólica se ha incrementado en un $18 \%$ respecto a 2009 .

La producción eólica de 2010 supera a la obtenida ese año por gran hidráulica (régimen ordinario) que, en un año de elevada hidraulicidad, genera $38.738 \mathrm{GWh}$; y se aproxima a la producción total hidroeléctrica. Esta suma a la anterior la obtenida en minicentrales (régimen especial) que hasta noviembre de 2010 asciende a $5.912 \mathrm{GWh}$, por lo que el total aproximado de 2010 es de 44.650 GWh, la cifra es la más elevada en las dos últimas décadas. Teniendo en cuenta que la producción hidroeléctrica solo ha superado los 40.000 GWh los años 1996, 2001, 2003 y 2010 (Espejo y García, 2010), la energía eólica es ya la primera tecnología renovable productora de electricidad en España.

\section{Distribución geográfica de la potencia instalada}

Como consecuencia de su latitud geográfica la Península Ibérica se encuentra ubicada fundamentalmente en la zona perturbada por los vientos del oeste. El territorio ibérico se caracteriza por ser zona de paso de las diferentes masas de aire alógenas que la alcanzan, tanto marítimas como continentales (Capel, 1999). Dejando al margen los sectores montañosos donde el venteo es continuo y si acontecen ventoleras más intensas, en España los sectores más afectados por los vientos a gran velocidad tienen una distribución anárquica y ello está en función de disposiciones de línea de costa y situación de relieves próximos proclives a la formación de pasillos o ventanías (Olcina, 1994). Por tanto, se distinguen:

- Litoral cantábrico y dentro de él la costa gallega entre la Ría de Arosa y la Estaca de Bares, y el sector ovetense en torno al Cabo de Peñas.

- Valle del Ebro, con un área de mayores velocidades del viento alrededor de la capital zaragozana.

- Castilla y León, en una franja extendida entre el suroeste de Burgos y noreste de Salamanca.
- La Mancha, y en particular la provincia de Albacete.

- Litoral mediterráneo con algunas ventanías: litoral gerundense (El Ampurdán), litoral levantino (entre Valencia y el Cabo de San Antonio) y la franja litoral de la Sierra del cabo de Gata (Almería).

- El cuadrante suroccidental de España, entre las bahías de Algeciras y Cádiz.

En las Islas Canarias el dominio, casi constante, de un cinturón de altas presiones dinámicas da lugar a una persistencia de los vientos alisios. Estos vientos, procedentes del flanco oriental del anticiclón de las Azores, poseen una gran regularidad anual. Este hecho y que en los lugares abiertos a estos vientos la frecuencia de las velocidades comprendidas entre los 25 y 40 kilómetros/hora $(\mathrm{km} / \mathrm{h})$ se acerque al $50 \%$, junto a la poca frecuencia de los superiores a $50 \mathrm{~km} / \mathrm{h}$ y a que el recorrido medio del viento sea del orden de 20 $\mathrm{km} / \mathrm{h}$, hace que estos lugares resulten idóneos para la utilización de la energía eólica (Font, 2000).

Para que la energía eólica pueda ser utilizada con cierta eficacia en una zona determinada, las características del viento deben cumplir una serie de condiciones relativas a velocidad, continuidad, estabilidad, etc. Un dato de gran importancia al respecto es la "densidad de potencia" del viento, es decir, el valor máximo de la potencia que se puede conseguir por cada unidad de área barrida por el viento. En concreto por debajo de los 50 vatios $/ \mathrm{m}^{2}$ no tiene interés el emplazamiento de instalaciones eólicas; y solo por encima de los 200 vatios $/ \mathrm{m}^{2}$ comienzan a resultar auténticamente rentables. Aun así hay que tener en cuenta que hacen falta densidades de potencia del viento superiores a 1.000 vatios/ $\mathrm{m}^{2}$ para que los aerogeneradores tengan un rendimiento aceptable.

El desarrollo de la energía eólica no se ha dado de igual modo en todo el territorio de España (Figura № 1). Las Comunidades Autónomas con mayor potencia eólica instalada son Castilla y León, Castilla-La Mancha, Galicia, Andalucía y Aragón. En cambio en Extremadura y la Comunidad de Madrid no hay un solo aerogenerador.

En Castilla y León, la producción energética tiene una larga tradición. En las dos últimas décadas, este carácter se ha reafirmado al participar activamente en el proceso de implantación de la producción eléctrica a partir de fuentes renovables, particularmente de 
origen eólico y solar. El Plan Eólico de Castilla y León busca la ordenación de la instalación de parques en el territorio para compatibilizar el aprovechamiento de los recursos con su desarrollo sostenible. Este Plan establece unos parámetros que conjugan viabilidad técnica y ambiental para determinar las ubicaciones en función de su capacidad de acogida, definida tanto en términos de potencialidad del recurso -continuidad e intensidad del vientocomo de evacuación de la producción (Baraja y Herrero, 2010).

Castilla-La Mancha se incorpora a la producción energética eólica en 1999, con una potencia instalada de $112 \mathrm{MW}$. Entre agosto de 1999 y septiembre de 2000 se ponen en marcha cinco parques eólicos en Higueruela (Albacete), que suman un total de 161,2 MW, convirtiéndose en el conjunto eólico más potente del mundo en ese momento. De aqueIlos $112 \mathrm{MW}$ ha pasado a $3.709 \mathrm{MW}$, por lo que ha multiplicado su capacidad por 33, en solo 11 años.

El Decreto 58/1999 de la Junta de Comunidades de Castilla-La Mancha desarrolla los procedimientos, condiciones y criterios que han de regir para la autorización de instalaciones de generación eléctrica a partir de energía eólica. También regula los Planes Eólicos Estratégicos, que comprenden la planificación por un mismo promotor y mediante inversiones plurianuales, de la implantación de dos o más parques eólicos, con el objeto de que la administración regional pueda identificar y valorar el contenido innovador del sector, la incidencia en la planificación energética sectorial y el desarrollo armónico de los aprovechamientos eólicos, así como su impacto sobre el tejido industrial y el desarroIlo económico local y regional, minimizando el impacto ambiental global de este tipo de actividad (Espejo, 2010).

En Aragón, entre 1996 y 2007, los parques eólicos han ejercido un impacto total sobre el conjunto de la economía que puede cifrarse en 1.777 millones de euros constantes de 2009. El volumen de empleo generado por la inversión durante este periodo ha sido, en promedio, de unos 1.183 puestos de trabajo anuales (no acumulativos). En cuanto al impacto ambiental, el coste evitado por la generación eólica en utilización de derechos se situó en 90,9 millones de euros en 2008. Adicionalmente, la producción eólica regional en esos años evitó la importación de 844.104 toneladas equivalentes de petróleo y supuso un ahorro de 130,4 millones de euros. La inversión en energía eólica también ha tenido un efecto notable sobre los presupuestos de los municipios en los que se ubican los parques eólicos $y$, adicionalmente, a través del canon por arrendamiento de los terrenos, que ha elevado las rentas privadas y públicas de esos municipios (Simón et al., 2009). El 22 de junio de 2010 el Consejo de Gobierno de la Diputación General de Aragón aprueba el Decreto $124 / 210$ por el que se regulan los procedimientos de priorización y autorización de producción de energía eléctrica a partir de la energía eólica, con una potencia superior a $100 \mathrm{~kW}$ e igual o inferior a 50 MW. El Gobierno de Aragón estima que esta nueva etapa establece más de $1.000 \mathrm{MW}$ adicionales de energía eólica, que en ese momento ya supera la mitad de la capacidad instalada en renovables.

Navarra es otra de las regiones pioneras en el desarrollo de la energía eólica. En esta Comunidad se ha generado, fruto de una política energética específica, un sector empresarial competitivo, con un importante impacto en términos de empleo de alta cualificación. A escala regional ha sido posible compatibilizar la preocupación medioambiental con el crecimiento económico y la creación de empleo (Pintor et al., 2006).

La Agrupación Empresarial InnovadoraClúster de Empresas de Energías Renovables, Medio Ambiente y Recursos Hídricos de las Islas Canarias publica en 2010 el Análisis de los sobrecostes de la Energía en el Sistema Energético de Canarias. En él se pone de manifiesto que la producción de electricidad con energía eólica en las islas es menos costosa que con otras tecnologías. De ahí que el Gobierno de España, a través del Real Decreto 1614/2010, haya establecido como objetivo de potencia eólica para esta Comunidad 600 MW, más de cuatro veces de la que cuenta en la actualidad. 
Figura $N^{\circ} 1$

Potencia eólica instalada en España (MW), 2010

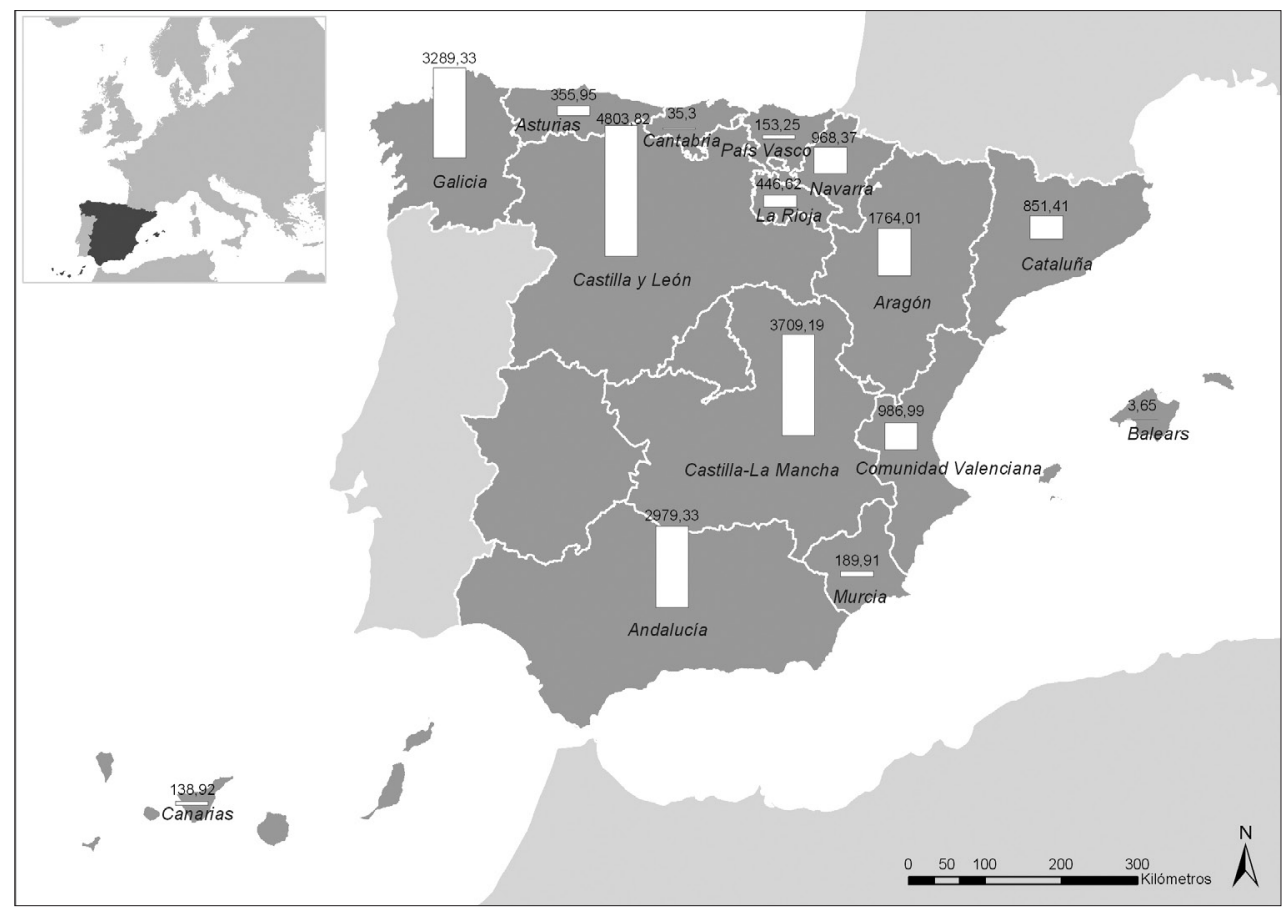

Fuente: Asociación Empresarial Eólica, 2010.

\section{La industria eólica española, un referente mundial}

Según el Estudio Macroeconómico del Impacto del Sector Eólico en España, realizado por Deloitte para la Asociación Empresarial Eólica (2009), el sector eólico aportó en 2009 al Producto Interior Bruto 3.207 millones de euros, que sumados a los dos años anteriores ascienden a 6.197 millones de euros, periodo en el que las primas percibidas por la eólica sumaron algo más de la mitad, 3.706 millones de euros. En 2009 el sector daba empleo, directa e indirectamente, a 35.719 personas. Continuó ahorrándole dinero a la economía española, ya que la sustitución de importaciones de combustibles fósiles ascendió a 1.541 millones de euros. Se ahorraron 270 millones por las emisiones de efecto invernadero evitadas y además el sector eólico exportó por valor de 2.104 millones, aportó 213 millones a la balanza fiscal e invirtió 156 millones en I+D+i (Deloitte, 2010).
El desarrollo inicial de la industria eólica se debe a pequeñas empresas que hacían sus propios diseños de aerogeneradores y compraban en el mercado los distintos componentes, para ensamblarlos primero en naves de montaje y posteriormente en talleres más especializados. Se fueron desarrollando así lazos de colaboración entre diseñadores y fabricantes de componentes, a la vez que se incrementaba el volumen de trabajo de estos últimos, que también eran pequeñas empresas. Poco a poco algunos fabricantes fueron especializándose o creando algún taIler con dedicación mayoritaria a la industria eólica. En España se ha ido configurando una industria de aerogeneradores cada vez más potente, que se mueve en un mercado internacional de creciente competitividad. Estas empresas han evolucionado al ritmo de la potencia instalada en energía eólica.

La Asociación Empresarial Eólica considera que la cadena de valor de la industria 
eólica en España se puede dividir en cuatro grandes subsectores: promotores de parques eólicos/productores de energía, fabricantes de aerogeneradores, fabricantes de componentes específicos (e.g. rodamientos, multiplicadoras) y servicios anexos (e.g. ingeniería y consultoría, servicios financieros, soluciones tecnológicas). La apuesta realizada por la eólica en España ha propiciado que se desarrollen en nuestro país todos los sectores de la cadena de valor, incluyendo empresas líderes a nivel mundial en cada uno de ellos. Más de cuarenta empresas del sector han salido de nuestras fronteras y se han instalado en los principales mercados mundiales, principalmente en Europa, Latinoamérica, Estados Unidos, Australia y China.
Además de ser cuantitativa, esta valoración es cualitativa, ya que en muchos casos, dichas empresas han tenido resultados destacados, siendo líderes en la instalación de potencia y producción de energía, la fabricación de equipos y la oferta de servicios auxiliares. También, el desarrollo de la potencia instalada a nivel nacional ha actuado como un incentivo a las empresas extranjeras para instalarse en España (Cuadro $N^{\circ} 3$ ), con el objetivo de aprovechar las ventajas competitivas que ofrece nuestro país: alta cualificación de los trabajadores, mercado en desarrollo, facilidades para el transporte y modelos de retribución adecuados a este tipo de energía (Deloitte, 2010).

Cuadro $N^{\circ} 3$

Centros de producción relacionados con la industria eólica en España, 2010

\begin{tabular}{|l|l|l|}
\hline EmPresa & Clasificación & Provincia \\
\hline 3M ESPAÑA S.A. & Generadores y componentes eléctricos & Madrid \\
ACCIONA BLADES & Palas & Navarra \\
ACCIONA WIND POWER & Palas & Toledo \\
Ensamblaje de aerogeneradores & Navarra \\
Castellón \\
ALSTOM POWER SERVICE, S.A. & Generadores y componentes eléctricos & Álava \\
ALSTOM WIND & Fabricación de torres de aerogeneradores. & Zamora \\
AREVA T\&D IBÉRICA, S.A & Ensamblaje de aerogeneradores & La Coruña \\
ASEA BROWN BOVERI, S.A. & Gistemas de control & Gavarra \\
AVANTI WIND SYSTEMS, S.L. & Generadores y componentes eléctricos & Madrid \\
C.C. JENSEN IBÉRICA, S.L. & Torres y componentes mecánicos & Madrid \\
COASA & Torres y componentes mecánicos & Zaragoza \\
COIPER & Palas & Ourense \\
COMPAÑÍA EÓLICA TIERRAS & Torres y componentes mecánicos & León \\
ALTAS S.A. & Mantenimiento integral de parques eólicos \\
CORUÑESA DE COMPOSITES & Ensamblaje de aerogeneradores & Soria \\
DANIGAL & Palas & La Coruña \\
DANOBATGROUP S. COOP. & Maquinaria & La Coruña \\
DIMECO & Torres y componentes mecánicos & Guipúzcoa \\
\hline
\end{tabular}




\begin{tabular}{|c|c|c|}
\hline Empresa & Clasificación & Provincia \\
\hline ELEVADORES GOIAN & Torres y componentes mecánicos & Guipúzcoa \\
\hline EMESA & Torres y componentes mecánicos & La Coruña \\
\hline ENERGEA & Generadores y componentes eléctricos & $\begin{array}{l}\text { Lugo } \\
\text { Pontevedra } \\
\text { La Coruña }\end{array}$ \\
\hline ENFLO WINTEC IBÉRICA & Ensamblaje de aerogeneradores & Navarra \\
\hline EOZEN & $\begin{array}{l}\text { Ensamblaje de aerogeneradores } \\
\text { Palas }\end{array}$ & Granada \\
\hline FIBERBLADE NORTE II & Torres y componentes mecánicos & La Coruña \\
\hline FLUITECNIK & Torres y componentes mecánicos & Navarra \\
\hline GALOL, S.A. & Recubrimiento de piezas & Valencia \\
\hline GAMESA & $\begin{array}{l}\text { Torres y componentes mecánicos } \\
\text { Ensamblaje de aerogeneradores } \\
\text { Generadores y componentes eléctricos } \\
\text { Palas } \\
\text { Multiplicadoras }\end{array}$ & $\begin{array}{l}\text { Jaén } \\
\text { Zaragoza } \\
\text { Asturias } \\
\text { Cantabria } \\
\text { Albacete } \\
\text { Cuenca } \\
\text { Soria } \\
\text { Valladolid } \\
\text { Burgos } \\
\text { La Coruña } \\
\text { Madrid } \\
\text { Navarra } \\
\text { Guipúzcoa } \\
\text { Vizcaya } \\
\text { Valencia }\end{array}$ \\
\hline GANOMAGOGA & Torres y componentes mecánicos & Pontevedra \\
\hline GE WIND ENERGY S.L. & Ensamblaje de aerogeneradores & Toledo \\
\hline GLUAL HIDRAULICA & Componentes Oleohidráulicos & Guipúzcoa \\
\hline GRUPO EYMOSA-VENTOGAL & Ensamblaje de aerogeneradores & La Coruña \\
\hline HORTA COSLADA & Torres y componentes mecánicos & Soria \\
\hline IM FUTURE, S.L. & Palas & La Coruña \\
\hline INDAR ELECTRIC, S.L. & Generadores y componentes eléctricos & Guipúzcoa \\
\hline INDRA & Servicios Logísticos & $\begin{array}{l}\text { Cádiz } \\
\text { León } \\
\text { La Coruña } \\
\text { Madrid }\end{array}$ \\
\hline INGETEAM PANELES, S.A. & Generadores y componentes eléctricos & Navarra \\
\hline INGETEAM SERVICE, S.A & $\begin{array}{l}\text { Operación y Mantenimiento de parques } \\
\text { eólicos }\end{array}$ & $\begin{array}{l}\text { Albacete } \\
\text { Lugo }\end{array}$ \\
\hline INNEO TORRES & Torres y componentes mecánicos & Toledo \\
\hline INTORD, S.A. & Torres y componentes mecánicos & Madrid \\
\hline
\end{tabular}




\begin{tabular}{|c|c|c|}
\hline KINTECH INGENIERIA, S.L. & Generadores y componentes eléctricos & Zaragoza \\
\hline LASO ABNORMAL LOADS S.A. & Transporte material eólico & Badajoz \\
\hline LM GLASFIBER EÓLICA, S.A. & Palas & Castellón \\
\hline LM GLASFIBER ESPAÑOLA, S.A. & Palas & León \\
\hline MAECO EÓLICA & $\begin{array}{l}\text { Mantenimiento, Correctivos, retrofit, } \\
\text { repuestos }\end{array}$ & $\begin{array}{l}\text { Ávila } \\
\text { Soria } \\
\text { Lugo } \\
\text { La Rioja }\end{array}$ \\
\hline $\begin{array}{l}\text { MANUFACTURAS ELÉCTRICAS } \\
\text { S.A.U. }\end{array}$ & Generadores y componentes eléctricos & Vizcaya \\
\hline MATZ-ERREKA S.COOP. & Torres y componentes mecánicos & Guipúzcoa \\
\hline $\begin{array}{l}\text { MECHANICAL LINKAGE } \\
\text { SOLUTIONS S.L. }\end{array}$ & Sistemas de control & Valladolid \\
\hline MONTAJES DEL ATLÁNTICO & Torres y componentes mecánicos & La Coruña \\
\hline M-TORRES & Ensamblaje de aerogeneradores & Soria \\
\hline NAVANTIA & Ensamblaje de aerogeneradores & La Coruña \\
\hline RONAUTICA RENOVABLES & Palas & Pontevedra \\
\hline $\begin{array}{l}\text { SANTOS MAQUINARIA } \\
\text { ELÉCTRICA, S.L. }\end{array}$ & Generadores y componentes eléctricos & Madrid \\
\hline SSB & Operación y Mantenimiento & $\begin{array}{l}\text { Madrid } \\
\text { Murcia }\end{array}$ \\
\hline TECNOARANDA & Torres y componentes mecánicos & Burgos \\
\hline TRÁCTEL IBÉRICA S.A. & Torres y componentes mecánicos & $\begin{array}{l}\text { Huesca } \\
\text { Barcelona }\end{array}$ \\
\hline VESTAS BLADES SPAIN, S.L.U. & Palas & Ciudad Real \\
\hline $\begin{array}{l}\text { VESTAS CONTROL SYSTEMS } \\
\text { SPAIN, S.L. }\end{array}$ & Generadores y componentes eléctricos & Soria \\
\hline $\begin{array}{l}\text { VESTAS NACELLES SPAIN, } \\
\text { S.A.U. }\end{array}$ & Ensamblaje de aerogeneradores & $\begin{array}{l}\text { León } \\
\text { Lugo }\end{array}$ \\
\hline VOITH TURBO, S.A. & Torres y componentes mecánicos & Madrid \\
\hline ZF SERVICES ESPAÑA, S.A.U. & Multiplicadoras & Madrid \\
\hline
\end{tabular}

Fuente: Asociación Empresarial Eólica, 2010.

Entre las empresas españolas destaca Gamesa, nacida en 1976 como una empresa innovadora en el ámbito tecnológico. Entra en el sector de las renovables con una licencia tecnológica para fabricar aerogeneradores y en unos años consigue desarrollar tecnología propia y exportarla a Europa y América. Actualmente tiene su fabricación distribuida en Europa, Estados Unidos y China, y comercia- liza sus productos por todo el mundo (López, 2007; López-Vico, 2008). El Plan Estratégico de Gamesa 2011-2013, presentado en Londres en octubre de 2010, prevé invertir 250 millones durante estos años, y como vectores estratégicos tiene la reducción del coste de energía, el crecimiento y la mejora de la eficiencia. 
A finales de 2009 Gamesa, con 19.925 MW, ocupa el sexto puesto a nivel mundial en potencia eólica instalada, con un $12 \%$. Esta participación se cuadruplica en España, donde acumula casi la mitad de la capacidad (Cuadro $\mathrm{N}^{\circ} 4$ ), le sigue en importancia Vestas, la multinacional danesa que en 2009 ocupa el primer puesto mundial con $39.705 \mathrm{MW}$.

Desde noviembre de 2010 Gamesa coordina, junto con otras 10 empresas y 22 centros de investigación, el proyecto Azimut. Energía Eólica Offshore 2020. En el mismo participan también Alstom Wind, Acciona Windpower, Iberdrola Renovables y Acciona Energía; así como Técnicas Reunidas, Ingeteam, Ingeciber, Imatia, Tecnitest Ingenieros y DIgSILENT Ibérica.

El proyecto Azimut, aprobado por el Centro para el Desarrollo Tecnológico Industrial (CDTI) del Ministerio de Ciencia e Innovación, en el marco de la sexta convocatoria de ayudas a la I+D del Programa CENIT (Consor- cios Estratégicos Nacionales en Investigación Técnica), requerirá una inversión total de 25 millones de euros en los próximos cuatro años, cofinanciado por las compañías participantes. La iniciativa, cuya culminación se estima en 2013, pretende sentar las bases tecnológicas para el desarrollo posterior de un aerogenerador offshore de gran tamaño, previsto para 2020. Se han establecido como objetivos preliminares conseguir una potencia unitaria de $15 \mathrm{MW}$, así como superar las barreras técnicas y económicas que limitan en la actualidad el despliegue de la energía eólica marina. Entre estas, destacan la disponibilidad, las cimentaciones y la evacuación de energía a tierra, con el fin de acercar el coste de la energía eólica marina al de los emplazamientos en tierra. Las empresas participantes refuerzan así su apuesta por la I+D en energía eólica marina, como vía para alcanzar el liderazgo tecnológico mundial y contribuir así a una generación de energía renovable fiable, económica y con el máximo respeto al medio ambiente.

\section{Cuadro $\mathrm{N}^{\circ} 4$}

Potencia instalada por fabricantes, 2010

\begin{tabular}{|l|r|r|}
\hline Fabricante & Potencia MW & $\begin{array}{r}\text { Porcentaje de cuota de } \\
\text { mercado }\end{array}$ \\
\hline GAMESA & $9.846,34$ & 47,62 \\
VESTAS & $3.528,72$ & 17,07 \\
ALSTOM-WIND & $1.559,85$ & 7,54 \\
ACCIONA WIND POWER & $1.455,15$ & 7,04 \\
MADE & $1.261,73$ & 6,10 \\
GENERAL ELECTRIC & $1.203,20$ & 5,82 \\
SIEMENS & 727,10 & 3,52 \\
ENERCON & 484,60 & 2,34 \\
SUZLON & 218,00 & 1,05 \\
NORDEX & 135,18 & 0,65 \\
DESA & 101,02 & 0,49 \\
LAGERWEY & 35,70 & 0,17 \\
M-TORRES & 36,90 & 0,18 \\
KENETECH & 36,90 & 0,18 \\
REPOWER & 25,00 & 0,12 \\
FUHRLANDER & 12,00 & 0,06 \\
OTROS & 8,65 & 0,04 \\
Total & $20.676,05$ & 100 \\
\hline
\end{tabular}

Fuente: Asociación Empresarial Eólica, 2010. 
Iberdrola Renovables, Acciona Energía y Electricidade de Portugal Renováveis (EDPR) son las principales promotoras de la energía eólica en España (Cuadro № 5), y están presentes en los países en los que más se ha desarrollado esta tecnología de producción eléctrica (Cuadro $N^{\circ}$ 6).

\section{Cuadro $\mathrm{N}^{\circ} 5$}

Empresas que han promovido la energía eólica en España, 2010

\begin{tabular}{|l|r|r|}
\hline Promotor & Potencia MW & $\begin{array}{r}\text { Porcentaje de cuota de } \\
\text { mercado }\end{array}$ \\
\hline IBERDROLA RENOVALES & $5.168,50$ & 25,00 \\
ACCIONA ENERGÍA & $4.036,82$ & 19,52 \\
EDPR & $1.862,92$ & 9,01 \\
EUFER & 913,78 & 4,42 \\
ENEL GREEN POWER ESPANA & 807,51 & 3,91 \\
EYRA & 774,26 & 3,74 \\
OLIVENTO & 421,79 & 2,04 \\
ENERFÍN & 404,54 & 1,96 \\
AERSA & 390,69 & 1,89 \\
GAS NATURAL & 380,14 & 1,84 \\
EOLIA RENOVABLES & 367,74 & 1,78 \\
E.ON RENOVABLES & 321,33 & 1,55 \\
GAMESA ENERGÍA & 280,95 & 1,36 \\
MEDWIND & 245,25 & 1,19 \\
MOLINOS DEL EBRO & 235,16 & 1,14 \\
GOVADE & 232,52 & 1,12 \\
GECALSA & 231,06 & 1,12 \\
IBEREÓLICA & 158,90 & $0.676,05$ \\
EÓLICA DE NAVARRA & 149,11 & 0,77 \\
FERSA & 141,88 & 0,72 \\
ELECDEY & 140,09 & 0,69 \\
RENOVALIA & 105,00 & 0,68 \\
OTROS & & 0,51 \\
Total & 2906,10 & 100 \\
\hline
\end{tabular}

Fuente: Asociación Empresarial Eólica, 2010. 
Cuadro $\mathrm{N}^{\circ} 6$

Países en los que actúan las empresas españolas, 2010

\begin{tabular}{|c|c|}
\hline Empresa & Países \\
\hline ACCIONA ENERGÍA & $\begin{array}{l}\text { Estados Unidos, Canadá, México, India, Corea del Sur, Alemania, } \\
\text { Italia, Portugal, Grecia, Hungría, Francia, Marruecos }\end{array}$ \\
\hline GAMESA & $\begin{array}{l}\text { Francia, Grecia, Portugal, Alemania, Italia, China, Estados } \\
\text { Unidos, México, Rumania, Bulgaria, Alemania, Polonia, Suecia, } \\
\text { Reino Unido }\end{array}$ \\
\hline IBERDROLA RENOVABLES & $\begin{array}{l}\text { Estados Unidos, Reino Unido, Francia, Grecia, Polonia, Hungría, } \\
\text { Portugal, México, Italia, Alemania, Brasil, Irlanda }\end{array}$ \\
\hline $\begin{array}{l}\text { ENDESA COGENERACIÓN Y } \\
\text { RENOVABLES }\end{array}$ & Chile, Portugal \\
\hline EUFER & Portugal \\
\hline GUASCOR WIND & Argentina \\
\hline FERSA & $\begin{array}{l}\text { Polonia, Estonia, Francia, Italia, India, China, Panamá y } \\
\text { Montenegro }\end{array}$ \\
\hline GESTAMP & $\begin{array}{l}\text { Brasil, Estados Unidos, Turquía, Polonia, Bélgica, Rumania, } \\
\text { Bulgaria }\end{array}$ \\
\hline EOLIA RENOVABLES & Alemania, Francia, Polonia. Portugal, México, Canadá \\
\hline PRENEAL & Hungría, Bulgaria, Grecia, Croacia, Canadá, México \\
\hline EDP RENOVABLES & Francia, Reino Unido, Italia, Rumania, Polonia, Bélgica \\
\hline NATURENER & Canadá, Estados Unidos \\
\hline TAIGA & Polonia \\
\hline
\end{tabular}

\section{La política de fomento de las energías renovables y la energía eólica}

Las políticas energéticas de los países desarrollados tienen tres objetivos básicos: aumento de la competitividad de la economía con mejora de la eficiencia energética, integración de los objetivos medioambientales, y seguridad en el abastecimiento. Como instrumentos de política energética para lograr estos objetivos se presentan la diversificación de fuentes energéticas y sus procedencias, la mejora de eficiencia en el uso de la energía y su conservación, la investigación y desarrollo de nuevas tecnologías, y la cooperación entre países. La creciente preocupación social por la protección del medio ambiente lleva a la regulación de numerosos aspectos tendentes a aminorar el impacto de las actividades energéticas sobre el mismo. Por ello se han diseñado nuevas estrategias en el sector, en particular, las relativas a las emisiones de gases de efecto invernadero.

La Ley 54/1997 del sector eléctrico establece los principios de un nuevo modelo de funcionamiento basado en la libre competencia. Se da una libertad efectiva en cuanto a la instalación de centrales generadoras, es decir, no se limita la entrada en el mercado a ninguna instalación por razones de política energética o determinaciones de la planificación. La Ley hace compatible este fundamento con la consecución de otros objetivos tales como la mejora de la eficiencia energética, la reducción del consumo y la protección del 
medio ambiente, necesarios en función de los compromisos adquiridos por España en la reducción de gases productores del efecto invernadero. Establece para su consecución la existencia de un régimen especial de producción de energía eléctrica, como régimen diferenciado del ordinario. En este último, el esquema regulador es el mercado de producción en que se cruzan ofertas y demandas de electricidad y donde se establecen los precios como consecuencia de su funcionamiento como mercado organizado.

La nueva Ley hace obligada la promulgación del Real Decreto 2818/1998, de 23 de diciembre, sobre producción de energía eléctrica por instalaciones abastecidas por recursos o fuentes de energía renovables, residuos y cogeneración, con el fin de adecuar el funcionamiento de dicho régimen a la nueva regulación e introducción a la competencia. Este Real Decreto impulsa el desarrollo de instalaciones de régimen especial mediante la creación de un marco favorable sin incurrir en situaciones discriminatorias que pudieran ser limitadoras de una libre competencia, aunque estableciendo situaciones diferenciadas para aquellos sistemas energéticos que contribuyan con mayor eficacia a los objetivos antes señalados. Con el fin de alcanzar ese logro se fija un sistema de incentivos temporales para aquellas instalaciones que requieren de ellos para situarse en posición de competencia en un mercado libre. Para las instalaciones basadas en energías renovables y de residuos, el incentivo establecido no tiene límite temporal debido a que se hace necesario internalizar sus beneficios medioambientales ya que, por sus especiales características y nivel tecnológico, sus mayores costes no les permite la competencia en un mercado libre.
Se pueden acoger al régimen especial establecido en este Real Decreto aquellas instalaciones cuya potencia no supera los 50 MW, a partir de cogeneración u otras formas de producción de electricidad asociadas a actividades no eléctricas, siempre que supongan un alto rendimiento energético, o en grupos donde se utilicen como fuente de energía primaria alguna de las energías renovables no consumibles, biomasa o cualquier tipo de biocarburante, o residuos no renovables o procedentes de los sectores agrícola, ganadero y de servicios, con una potencia instalada igual o inferior a $25 \mathrm{MW}$, cuando supongan un alto rendimiento energético. La producción en régimen especial está acogida a un régimen económico singular (Comisión Nacional de Energía, 2008).

El Plan de Fomento de las Energías Renovables 2000-2010, aprobado por el Consejo de Ministros de 30 de diciembre de 1999, de acuerdo con la Ley 54/1997 del Sector Eléctrico, establece el objetivo de desarrollo a alcanzar por cada una de las áreas de energías renovables para cubrir, en su conjunto, al menos el $12 \%$ del consumo en términos de energía primaria en España en el año 2010. Este objetivo responde a las políticas de fomento de las energías renovables en la Unión Europea desde 1997, año en que se aprueba el documento Energía para el futuro. Libro Blanco para una Estrategia y un Plan de Acción Comunitarios. El Plan planteaba como objetivo que en 2010 haya instalados en España $8.974 \mathrm{MW}$ en energía eólica, distribuidos como se expone en el Cuadro $N^{\circ} 7$. 
Cuadro $\mathrm{N}^{\circ} 7$

Objetivos en energía eólica de los Planes de Energías Renovables (MW)

\begin{tabular}{|l|r|r|r|r|}
\hline Comunidad Autónoma & $\begin{array}{r}\text { Plan de Fomento } \\
\text { de las Energías } \\
\text { Renovables 2000- } \\
2010\end{array}$ & $\begin{array}{c}\text { Plan de Energías } \\
\text { Renovables } \\
2005-2010\end{array}$ & $\begin{array}{c}\text { Potencia } \\
\text { instalada 2010 }\end{array}$ & $\begin{array}{c}\text { Porcentaje } \\
\text { del grado de } \\
\text { cumplimiento } \\
\text { del Plan }\end{array}$ \\
\hline Castilla y León & 850 & 2.700 & $4.803,82$ & 177,92 \\
Castilla-La Mancha & 400 & 2.600 & $3.709,19$ & 142,66 \\
Galicia & 2.500 & 3.400 & $3.289,33$ & 96,75 \\
Andalucía & 1.100 & 2.200 & $2.979,33$ & 135,42 \\
Aragón & 1.000 & 2.400 & $1.764,01$ & 73,50 \\
Comunidad Valenciana & 290 & 1.600 & 986,99 & 61,69 \\
Navarra & 635 & 1.400 & 968,37 & 69,17 \\
Cataluña & 425 & 1.000 & 851,41 & 85,14 \\
La Rioja & 100 & 500 & 446,82 & 89,36 \\
Asturias & 300 & 450 & 355,95 & 79,10 \\
País Vasco & 200 & 250 & 153,25 & 61,30 \\
Murcia & 300 & 400 & 189,91 & 47,48 \\
Canarias & 250 & 630 & 138,92 & 22,05 \\
Cantabria & 300 & 300 & 35,30 & 11,77 \\
Baleares & 50 & 50 & 3,65 & 7,30 \\
Extremadura & 225 & 50 & 0,00 & 0,00 \\
Madrid & 5.974 & 20.155 & $20.676,05$ & 102,59 \\
Total & & & 0,00 \\
\hline
\end{tabular}

Fuente: Instituto para el Ahorro y la Diversificación Energética en España, y Asociación Empresarial Eólica, 2010 .

El Real Decreto 436/2004, de 12 de marzo, establece la metodología para la actualización y sistematización del régimen jurídico y económico de la actividad de producción de energía eléctrica en régimen especial. Las instalaciones de energía eólica quedan incluidas en el grupo b.2., que se divide en dos subgrupos: b.2.1 para las instalaciones ubicadas en tierra y b.2.2 para las instalaciones ubicadas en el mar.

Su artículo 34 expone que cuando se alcancen los $13.000 \mathrm{MW}$ de potencia instalada, se procederá a la revisión de la cuantía de las tarifas, incentivos y primas expresadas en el mismo.
El modelo retributivo adoptado en España es una variación del sistema denominado REFIT (Renewable Energy Feed-in Tariffs = Tarifas de introducción de la energía renovable), que es el que prevalece en Europa. De acuerdo con este sistema, los generadores de electricidad renovable tienen derecho a vender toda su producción a la red eléctrica y a ser por ello retribuidos bien a un precio fijo o bien al precio horario del mercado eléctrico general, además de un incentivo fijo que refleja o compensa el valor ambiental de la producción renovable. España permite a los generadores de electricidad renovable optar por seguir una u otra variante, es decir, el precio 
fijo o el precio de mercado más el incentivo fijo (López Sako, 2009).

En julio de 2005 el Gobierno aprueba el Plan de Energías Renovables en España 2005-2010 (PER), que constituye la revisión del Plan de Fomento de las Energías Renovables en España 2000-2010 hasta entonces vigente. Con esta revisión trata de mantener el compromiso de cubrir con fuentes renovables al menos el $12 \%$ del consumo total de energía en 2010. Sin embargo, propone una distribución diferente de los esfuerzos por áreas, de manera que sea posible la consecución del objetivo global. También incorpora los otros dos objetivos indicativos adoptados con posterioridad al anterior Plan: $29,4 \%$ de generación eléctrica con renovables y 5,75\% de biocarburantes en transporte para el año 2010.

Según el PER los objetivos eólicos mantenidos hasta ahora se presumen fácilmente alcanzables con anterioridad al 2010, sobre todo teniendo en cuenta que la potencia media instalada en los tres años anteriores al de su elaboración ha superado los $1.600 \mathrm{MW}$ anuales, no existiendo en esos momentos síntoma de decaimiento. Considera este Plan que existen factores que propician un mayor impulso al desarrollo del sector eólico en España:

- Amplio potencial eólico todavía sin aprovechar.

- Normativa favorable, que ha permitido consolidar la confianza y el interés de los promotores.

- Sector industrial maduro con muy elevado nivel tecnológico y capacidad de fabricación nacional.

- La planificación de los gobiernos autonómicos soportan los objetivos planteados.

- La incorporación de mejoras tecnológicas en el comportamiento de los aerogeneradores frente a la red, permitirá un alto grado de penetración de la energía eóli$\mathrm{ca}$, sin afectar a la red de abastecimiento eléctrico.

Se establece como objetivo eólico del PER, la instalación para el conjunto de España de una potencia eólica incremental de 12.000 MW en el periodo 2005-2010. Como se presenta en la tabla 7 , se realiza un desglose meramente indicativo, y en ningún caso limitativo, de estos $12.000 \mathrm{MW}$ en cada una de las Comunidades. Para realizar esta distribución se tienen en cuenta los siguientes factores: recursos eólicos existentes, grado de desarrollo regional de la energía eólica (instalaciones en funcionamiento, en construcción y en fase de tramitación administrativa), la planificación eólica de las Comunidades Autónomas, el estado de la normativa regional y su grado de desarrollo, y la evolución tecnológica territorial en el sector eólico. Como se comprueba en la tabla 7 el objetivo para el conjunto de España se ha cumplido, aunque con resultados distintos para cada Comunidad.

En relación a las instalaciones eólicas ubicadas en el mar, cuando se redacta el PER no hay ninguna, aunque existen diversos proyectos eólicos marinos ambiciosos, y si se superan las barreras para su implantación, cabría pensar que en el horizonte del año 2010, estas instalaciones podrían aportar en torno a $1.000 \mathrm{MW}$, hecho que no ha sucedido.

El Boletín Oficial del Estado de 24 de octubre de 2006 publica la Resolución de 4 de octubre de 2006, de la Secretaría General de Energía, por la que se aprueba el procedimiento de operación que establece los requisitos que han de cumplir las instalaciones de producción en régimen especial a efectos de garantizar la continuidad en el suministro frente a huecos de tensión, en cumplimiento de lo establecido en la disposición adicional cuarta del R.D. 436/2004. Su ámbito de aplicación es para instalaciones que utilicen la energía eólica como fuente exclusiva de energía primaria. Este procedimiento es de aplicación para los nuevos parques eólicos que se conecten al sistema eléctrico y cuya fecha de inscripción definitiva sea posterior a 1 de enero de 2007.

El Real Decreto 661/2007, de 25 de mayo, regula la actividad de producción de energía eléctrica en régimen especial. Sustituye al Real Decreto 436/2004 y da una nueva regulación a la actividad de producción de energía eléctrica en régimen especial, manteniendo la estructura básica de la existente. Su marco económico garantiza a los titulares de estas instalaciones una retribución razonable para sus inversiones y a los consumidores 
finales eléctricos una asignación racional de los costes imputables al sistema eléctrico.

En su disposición transitoria séptima se contempla la repotenciación de instalaciones eólicas con fecha de inscripción definitiva anterior al 31 de diciembre de 2001. Estas instalaciones podrán sustituir sus aerogeneradores por otros de mayor potencia, en unas condiciones determinadas, y a esto se le denomina repotenciación. Se establece un objetivo límite de potencia de $2.000 \mathrm{MW}$ adicionales a la potencia instalada de las instalaciones susceptibles de ser repotenciadas. Para estas instalaciones, mediante acuerdo del Consejo de Ministros, previa consulta con las Comunidades Autónomas, podrá determinarse el derecho a una prima adicional, específica para cada instalación, máxima de 0,7 céntimos de euro/kWh, a percibir hasta el 31 de diciembre de 2017. Las instalaciones deberán estar adscritas a un centro de control de generación y deberán disponer de los equipos técnicos necesarios para contribuir a la continuidad de suministro frente a huecos de tensión, de acuerdo con los procedimientos de operación correspondientes, exigibles a las nuevas instalaciones.

En mayo de 2009 se publica el Real Decreto 6/2009, de 30 de abril, por el que se adoptan medidas en el sector energético y se aprueba el bono social. En él se plantea que se hace necesario adoptar una medida de urgencia que garantice la necesaria seguridad jurídica a aquellos que han realizado inversiones, y ponga las bases para el establecimiento de nuevos regímenes económicos que propicien el cumplimiento de los objetivos pretendidos: la consecución de unos objetivos de potencia por tecnología a un coste razonable para el consumidor y la evolución tecnológica de las mismas que permita una reducción gradual de sus costes y por consiguiente su convergencia con las tecnologías convencionales.

Se crea un Registro de Preasignación de Retribución, con el fin de conocer en los plazos previstos en este Decreto las instalaciones que, en su fecha de publicación, no solo están proyectadas, sino que cumplen las condiciones para ejecutarse y acceder a todos los requisitos legales y reglamentarios, el volumen de potencia asociado a las mismas y el impacto en costes de la tarifa eléctrica y su calendario. La inscripción en el Registro de Preasignación es condición necesaria para el otorgamiento del derecho al régimen económico establecido en el Real Decreto $661 / 2007$.

En el Boletín Oficial del Estado, de 24 de noviembre de 2009, se publica el Acuerdo del Consejo de Ministros de 13 de noviembre de 2009, por el que se procede a la ordenación de los proyectos o instalaciones presentadas en el Registro de Preasignación de retribuciones para las instalaciones de producción de energía eléctrica, previsto en el Real Decreto 6/2009.

El Consejo de Ministros acuerda disponer la puesta en funcionamiento de las instalaciones eólicas en fases sucesivas de acuerdo con el siguiente ritmo acumulado de implantación: Fase 1: $3.719 \mathrm{MW}$ para los años 2009 y 2010, Fase 2: 5.419 MW para 2011, y Fase 3: para 2012 resto de potencia inscrita al amparo de lo previsto en la disposición transitoria quinta del Real Decreto 6/2009.

El 2 de julio de 2010 el Ministerio de Industria cierra un pacto con la industria para recortar ayudas a las eólicas y termosolares. Se anuncia un recorte del $35 \%$ de la prima de referencia para la eólica hasta finales de 2012. Pero la medida solo afecta al $25 \%$ de la potencia, la instalada al amparo del RD $661 / 2007$.

El 19 de octubre de 2010 la Asociación Empresarial Eólica reúne por primera vez a todas las asociaciones de España, de ámbito estatal y autonómico. En la reunión se constata que en todas las Comunidades Autónomas la principal preocupación es la incertidumbre regulatoria que pesa sobre el sector. Desde las asociaciones se insiste en la importancia de que salga cuanto antes una normativa que recoja el acuerdo, que se refiere a las instalaciones eólicas en funcionamiento, para que puedan comenzar las negociaciones sobre el marco económico por el que se regirá el sector a partir de 2013.

Con el Real Decreto 1614/2010, de 7 de diciembre, se regulan y modifican determinados aspectos de carácter económico para las instalaciones de tecnologías eólicas y solar termoeléctrica. Esta nueva norma garantiza que los parques eólicos volverán a percibir 
los niveles de diciembre de 2010 a partir de 2013, y que las revisiones futuras de las tarifas no afectarán en ningún caso a las instalaciones en funcionamiento. También elimina el riesgo de una revisión retroactiva de las primas y limita a 2.589 horas al año el pago de la prima, pero solo en los años en los que la media nacional supere las 2.350 horas.

La Directiva 2009/28/CE del Parlamento Europeo y del Consejo, de 23 de abril de 2009, relativa al fomento del uso de energía procedente de fuentes renovables, fija como objetivos generales conseguir una cuota del $20 \%$ en energía procedente de fuentes renovables en el consumo final bruto de energía en la Unión Europea para el año 2020. La Directiva establece la necesidad de que cada Estado miembro elabore y notifique a la Comisión Europea, a más tardar del 30 de junio de 2010, un Plan de Acción Nacional de Energías Renovables (PANER) para el periodo 2011-2020, con vistas al cumplimiento de los objetivos vinculantes que fija la Directiva

En el documento del PANER se indica que en esos momentos persisten diversos factores que propician un mayor desarrollo eólico del actual en el horizonte 2020, entre los que destacan tanto la existencia de un amplio potencial eólico todavía sin aprovechar, tanto en tierra como mar adentro -plasmado en los ambiciosos objetivos que contemplan las planificaciones energéticas autonómicas-, como los esfuerzos del Operador del Sistema Eléctrico Español (Red Eléctrica de España) y de la industria eólica -mediante la incorporación de nuevas herramientas de gestión, requisitos de operación y continuas mejoras tecnológicas-, para maximizar la penetración eólica en el sistema.

Diferenciando según tecnologías de energías renovables, la energía eólica seguirá ocupando un lugar dominante, con el $52 \%$ de la producción eléctrica renovable en 2020 considerando conjuntamente la terrestre y marina.

Se consideran los siguientes objetivos eólicos en el horizonte 2020: eólica en tierra: $35.000 \mathrm{MW}$ (34.630 en media y gran potencia, y 370 en pequeña potencia) y eólica marina $3.000 \mathrm{MW}$ (Cuadro $\mathrm{N}^{\circ}$ 8). A partir de 2015, se espera que comiencen a entrar en servicio parques eólicos marinos de gran potencia en el litoral español. En el horizonte 2020 se prevé que todos ellos se implanten a profundidades menores de $50 \mathrm{~m}$. Se espera un incremento anual de la potencia eólica marina instalada progresivo hasta los 750 MW en 2020, de manera que finalice ese año con unos 3.000 MW eólicos marinos.

\section{Cuadro $\mathrm{N}^{\circ} 8$}

Objetivos de potencia y generación de energía eólica establecidos en el Plan de Acción Nacional en Materia de Energías Renovables, 2010-2020

\begin{tabular}{|c|c|c|c|c|c|c|}
\hline \multirow[t]{2}{*}{ Año } & \multicolumn{3}{|c|}{$\begin{array}{c}\text { Potencia } \\
\text { MW }\end{array}$} & \multicolumn{3}{|c|}{$\begin{array}{c}\text { Producción } \\
\text { GWH }\end{array}$} \\
\hline & En tierra & Mar adentro & Total & En tierra & Mar adentro & Total \\
\hline 2010 & 20.155 & 0 & 20.155 & 40.978 & 0 & 40.978 \\
\hline 2011 & 21.855 & 0 & 21.855 & 43.668 & 0 & 43.668 \\
\hline 2012 & 23.555 & 0 & 23.555 & 47.312 & 0 & 47.312 \\
\hline 2013 & 24.986 & 0 & 24.986 & 50.753 & 0 & 50.753 \\
\hline 2014 & 26.466 & 50 & 26.516 & 53.906 & 75 & 53.981 \\
\hline 2015 & 27.847 & 150 & 27.997 & 56.786 & 300 & 57.086 \\
\hline 2016 & 29.778 & 500 & 30.278 & 59.598 & 975 & 60.573 \\
\hline 2017 & 30.708 & 1.000 & 31.708 & 62.238 & 2.245 & 64.483 \\
\hline 2018 & 32.139 & 1.500 & 33.639 & 64.925 & 3.727 & 68.652 \\
\hline 2019 & 33.569 & 2.250 & 35.819 & 67.619 & 5.577 & 73.196 \\
\hline 2020 & 35.000 & 3.000 & 38.000 & 70.502 & 7.753 & 78.255 \\
\hline
\end{tabular}

Fuente: Plan de Acción Nacional de Energías Renovables en España, 2011-2020. 


\section{Las ampliación de las redes eléctricas para el óptimo aprovechamiento de las energías renovables}

Entre los retos a los que tienen que dar respuesta los operadores de redes eléctrica están el de acomodar en nuestro sistema eléctrico las energías renovables, muy repartidas por el territorio, en ocasiones muy alejadas de los centros de consumo, con una gran variabilidad en su aportación en función de las condiciones meteorológicas y, por tanto, con menor firmeza a la hora de asegurar el equilibrio instantáneo entre la producción y el consumo, que es el principio fundamental de la estabilidad de los sistemas eléctricos.

En 2010 la energía eólica superó en varias ocasiones los anteriores máximos históricos de potencia instantánea, de energía horaria y de energía diaria. El 9 de noviembre se registró el último récord de energía diaria con $315.258 \mathrm{MWh}$, una producción que permitió cubrir el $43 \%$ de la demanda de ese día. Asimismo, en febrero se produjo un máximo mensual de energía eólica que cubrió el $21 \%$ de la demanda de ese mes. Sin embargo, la variabilidad que caracteriza esta energía ha dado lugar a situaciones extremas como la producida el mismo día 9 de noviembre $(3,35$ horas) en la que el $54 \%$ de la demanda fue cubierta con esta energía, mientras que el día 26 de junio a las 10.32 horas apenas cubrió el 1\% (Red Eléctrica de España, 2011).

Los operadores de redes eléctricas tienen que suministrar toda la energía demandada, en todo momento, sin excepción, con viento y sin viento, de día y de noche, con calor o con frío, en años secos y en años húmedos. Por ello son fundamentales las inversiones en la red de transporte. Hay que desarrollar una red de gran capacidad de carácter internacional en convivencia con una red más robusta, flexible y mallada de carácter nacional, con capacidad para gestionar y compensar flujos que varían considerablemente en su origendestino en función de las condiciones meteorológicas. Esa red de transporte de gran capacidad facilitará el aprovechamiento en cada momento de la generación económicamente más eficiente, en beneficio de la competiti- vidad de las empresas y el poder adquisitivo de las familias. Para conseguir esa red es necesario eliminar los prejuicios, restricciones y barreras que frenan su desarrollo y amenazan con limitar la contribución del sistema eléctrico a un suministro eficiente, limpio y seguro (Atienza, 2010).

España dispone de 100.000 MW de potencia instalada para la producción de eléctrica, de los que más del $20 \%$ son de energía eólica. En cambio, la capacidad de exportación de electricidad hacia Europa, a través de Francia, no llega al 2\% de esa potencia. Esta situación está limitando la exportación de los excedentes de producción eléctrica eólica que se dan en determinados momentos a lo largo del año, y que obliga en ocasiones a paralizar los aerogeneradores para que no produzcan electricidad que no se consume.

La ampliación de la red de transporte internacional también en necesaria para el sector de producción eléctrica con centrales de ciclo combinado, que utilizan gas natural para la combustión (Espejo y Capel, 2007). Estas instalaciones suponen la garantía de abastecimiento de España, por eso hay quien afirma que cada vez que se inaugura un parque eólico, hay que contar con idéntica potencia en ciclo combinado. La falta de capacidad para exportar electricidad de esta procedencia también está afectando seriamente a este sector. En el año 2010 el coeficiente de utilización de los $25.000 \mathrm{MW}$ instalados en la España peninsular fue solo del $32 \%$, lo que significa que están funcionando por debajo de un tercio de su capacidad de producción.

\section{Conclusiones}

España cuenta con una larga tradición del aprovechamiento del viento como recurso energético, los molinos de viento durante siglos permitieron la molienda del grano en gran parte de su territorio. Desde mediados de los años noventa del siglo pasado ha apostado por la energía eólica y se ha convertido en una potencia mundial en el aprovechamiento de esta fuente energética.

La existencia de un marco normativo estable ha supuesto la instalación de más de 
20.000 MW de potencia de generación de esta tecnología, lo que ha permitido que en los últimos años aparezca una industria especializada en generación eólica de mucha relevancia con empresas entre las más importantes del mundo en este sector.

Según la Asociación Empresarial Eólica, esta energía recibió el año 20091.600 millones de euros en primas, pero evitó la importación de combustibles fósiles por valor de 3.000 millones.

La implantación de parques eólicos en áreas rurales con escaso desarrollo económico supone una gran fuente de ingresos para los municipios. Los ayuntamientos los obtienen con las licencias de obras, impuesto de actividades económicas y con la renta percibida cuando los aerogeneradores se instalan en terrenos de propiedad municipal. Los habitantes de estos territorios, además de alquilar sus tierras a las empresas con este fin, encuentran empleo durante el tiempo que dura la construcción, que dada la envergadura de la mayoría de ellos, se suele prolongar durante varios meses; una vez construidos se generan empleos para su mantenimiento.

El desarrollo de la energía eólica en España no parece que se vaya a detener por varias razones: la existencia de un potencial eólico significativo; la simplicidad a la hora de su implantación, ya que se trata de instalaciones modulares con equipos iguales y poca obra civil asociada; las posibilidades que ofrece de mejora técnica, aumento de potencia unitaria y reducción significativa de costes; por su reducido impacto ambiental en comparación con otras tecnologías de producción de energía eléctrica; y sobre todo por el importante apoyo político, tanto estatal como de la mayoría de las Comunidades Autónomas.

Para garantizar la distribución de la energía eólica y otras renovables, hay que desarrollar una red de gran capacidad de carácter internacional en convivencia con una red más robusta, flexible y mallada de carácter nacional, con capacidad para gestionar y compensar flujos que varían considerablemente en su origen-destino en función de las condiciones meteorológicas.

\section{Referencias bibliográficas}

ATIENZA, L. Redes eléctricas para un desarrollo sostenible. El País Negocios, 31 de octubre de 2010, p. 23.

BARAJA, E. y HERRERO, D. Energías renovables en Castilla y León: estudio de caso. Nimbus, 2010, № 25-26, p. 21-42.

CAPEL, J.J. La presión atmosférica y los vientos en la Península Ibérica. Reflexiones sobre el Monzón Ibérico. Nimbus, 1999, № 4, p. 5-60.

COMISIÓN NACIONAL DE ENERGÍA. LeY del Sector Eléctrico. Madrid, 2008.

DELOITTE. Estudio Macroeconómico del Impacto del Sector Eólico en España. Madrid: Asociación Empresarial Eólica, 2010.

DOMÍNGUEZ, J.; LAGO, C.; PRADES, A. y DÍAZ, M.P. Energías renovables y modelo energético, una perspectiva desde la sostenibilidad. Nimbus, 2010, № 25-26, p. 43-63.

ESPEJO, C. La energía eólica en España. Investigaciones Geográficas, 2004, № 35, p. 45-65.

ESPEJO, C. La energía eólica en CastillaLa Mancha: referente nacional e internacional. En: CEBRIÁN, F.; PILLET, F. y CARPIO, J. (Editores). Las escalas de la Geografía: del mundo al lugar. Homenaje al Profesor Miguel Panadero Moya. Cuenca: Universidad de Castilla-La Mancha, 2010, p. 175-196.

ESPEJO, C. y CAPEL, J.J. El gas en la producción de electricidad en España. Nimbus, 2007, No 19-20, p. 71-97.

ESPEJO, C. y GARCÍA, R. Agua y energía: producción hidroeléctrica en España. Investigaciones Geográficas, 2010, № 51, p. 107-129.

FONT, I. Climatología de España y Portugal. Salamanca: Universidad de Salamanca, 2000.

FROLOVA, M. Los paisajes de la energía eólica: su percepción social y gestión en España. Nimbus, 2010, № 25-26, p. 93-100. 
FROLOVA, M. y PÉREZ, B. El desarrollo de las energías renovables y el paisaje: algunas bases para la implementación de la Convención Europea del Paisaje en la Política Energética Española. Cuadernos Geográficos de la Universidad de Granada, 2008, № 43, p. 289-309.

GALDÓS, R. y MADRID, F.J. La energía eólica en España y su contribución al desarrollo rural. Investigaciones Geográficas, 2009, № 50, p. 93-108.

LÓPEZ, J.I. Energía eólica. En: MARTíNEZ-VAL, J.M. (Editor). La energía en sus claves. Madrid: Fundación Iberdrola, 2004, p. 371-395.

LÓPEZ, M.J. Regulación y autorización de los parques eólicos. Cizur Menor: Editorial Aranzadi, 2008.

LÓPEZ, M.J. Apoyo público a la energía eólica en España. Revista de Derecho, 2009, No 31, p. 224-255.

LÓPEZ, N. La internacionalización basada en la innovación. El caso de Gamesa. Economía Industrial, 2007, № 365, p. 179-192.

LÓPEZ-VICO, V. La internacionalización en el sector eólico. El caso de Gamesa. Boletín Económico del ICE, 2008, № 2.944, p. 15-30.

MARTíNEZ, A., BAYOD, A.A. y PÉREZ, M. La industria de la energía eólica en España. Tecnología y desarrollo rural endógeno. Boletín Económico del ICE, 2002, № 2.740, p. 19-29.

MÉRIDA, M.; PÉREZ, B.; LOBÓN, R. Y FROLOVA, M. Hacia la caracterización del paisaje de energías renovables. En: Geografía, territorio y paisaje: el estado de la cuestión. Cuenca: Universidad de Castilla-La Mancha, 2009, p. 1.193-1.210.
OLCINA, J. Riesgos climáticos en la Península Ibérica. Madrid: Libros Penthalon-Acción Divulgativa, 1994.

PÉREZ, B. Energía eólica marina y paisaje. Un futuro incierto. ENOVA. Energías limpias de Andalucía, 2009, № 2, p. 24-25.

PÉREZ, B. Perspectivas de desarrollo y ordenación territorial y paisajística de la energía eólica off-shore en España. Nimbus, 2010, No 25-26, p. 175-185.

PÉREZ, B.; REQUEJO, J. y BALLESTEROS, C. Energías renovables y paisaje: incidencia en el paisaje de parques eólicos y plantas fotovoltaicas. En: SÁNCHEZ, L. y TROITIÑO, M.A. (Coordinadores). Agua, territorio y paisaje: de los instrumentos programados a la planificación aplicada: $V$ Congreso Internacional de Ordenación del Territorio. Madrid: Asociación Interprofesional de Ordenación del Territorio. FUNDICOT, 2009, p. 1.1911.204.

PINTOR, J.M.; LERA, F.; GARCÍA, J. y FAULÍN, J. Energía eólica y empleo: el caso de Navarra como paradigma. Información Comercial Española, 2006, № 829, p. 253-271.

RED ELÉCTRICA DE ESPAÑA. El sistema eléctrico español. Avance del Informe 2010. Madrid, 2011.

SIMÓN, B.; AIXALÁ, J. y PÉREZ, L. Efectos económicos de la energía eólica en Aragón (1996-2012). Economía Aragonesa, 2009, № 40, p. 56-72. 